\title{
Component analysis of illicit morphia tablets (clandestine laboratory preparation) using gas chromatography mass spectrometry: a case study
}

Muhammad Usman * (D), Tahir Jamshaid, Abid Naseer, Yawar Baig, Zahid Mehmood, Muhammad Shahwar, Shahnaz Akhtar, Muhammad Taimoor Chaudhary, Muhammad Sarwar and Muhammad Ashraf Tahir

\begin{abstract}
Background: Various synthetic or semi-synthetic drugs like cocaine, amphetamine type stimulants (ATS), and heroin are produced and processed in clandestine laboratories. Every clan lab has its own procedures and protocols for drug synthesis and processing. A drug can be brought up in different forms like pharmaceutical tablets or raw street powder or any else, depending upon different types of adulterants or diluents and the physical modifications required for its transportation. Since a specific drug is synthesized by various clandestine laboratories, it is likely to have some differences between the two samples due to slightly different synthesis protocols used or the nature and concentration of adulterants. Detailed analysis of drug samples can determine, whether the drug belongs to common source or not. The basic purpose of alterations in physical form is to deceive the law enforcement agencies and to increase the bulk.
\end{abstract}

Case presentation: In this article, a clandestine product of an altered form of a drug was analyzed and reported. Two samples of orange colored "morphia tablets" seized by law enforcement officials were submitted for chemical analysis. Both of the samples were analyzed to determine physical and chemical characteristics. In both samples, the tablets were orange in color. The average weight of a tablet was $58.24 \mathrm{mg}$ for sample A, and $68.85 \mathrm{mg}$ for sample B. Qualitative analysis using GC-MS showed that tablets in sample A were composed of caffeine, dextromethorphan, hydromorphone, acetylcodeine, and heroin, while the tablets in sample B were composed of caffeine, acetylcodeine, heroin, papaverine, and noscapine. The concentration of heroin in sample B was much higher than that in sample A.

Conclusion: The analysis of morphia tablets showed that these tablets were an altered form of street heroin. On the basis of variation in weight, heroin concentration, impurities, and adulterants in both the samples analyzed, it can be concluded that they belonged to some different sources. Complete drug profiling for organic and inorganic components of tablets is recommended for future prospective.

Keywords: Forensic science, Forensic chemistry, Morphia tablets, Clandestine laboratory preparations, Street heroin, Adulterants, GC-MS, Narcotic/illicit drugs

\footnotetext{
* Correspondence: usman.iqba@pfsa.gop.pk; m.usman.iqbal9009@gmail.com

Narcotic Department, Punjab Forensic Science Agency, Old Multan Road,

Thokar Niaz Baig, Lahore 53700, Pakistan
} 


\section{Background}

Clandestine laboratories established by the criminals are involved in drug markets to synthesize and proliferate the abuse of controlled substances. Clandestine labs are not merely associated with the synthesis of drugs but also the explosives and chemical or biological weapons. These illegal clandestine laboratories have been found throughout the world (urban, rural, and metropolitan cities) to provide drugs of abuse to the illicit market. Some drugs of abuse reach black market by alternate trafficking routes from authentic sources in crude form (i.e., poppy from Afghanistan), while others are processed before the supply (i.e., garda from Cannabis and morphine from poppy resin). There are some other drugs with high potential for abuse and are easy to produce in clandestine laboratories like, cocaine, amphetamines, methamphetamine, and heroin. Easy and cheap production of illicit drugs combined with high-profit margins is the main source of motivation to establish a clandestine laboratory (Frank and Sobol, 1990; Sobol, 2012). In such premises, not only the illicit drugs are synthesized from precursors, but also street level drugs produced by adding cutting agents (adulterants or diluents) in a highly pure drug to increase the bulk (Sulaiman et al., 2017). Tablets of different shapes and colors are also synthesized in order to change/mask the general appearance of a drug, so that it can be trafficked easily in disguise and sold in drug market.

Pakistan is located in golden crescent (Syeda et al., 2017). Drug abuse and trafficking are among the major problems faced by the country, governed by its critical geographical location (Emmanuel and Attarad, 2006). Opium, cannabis, and heroin along with some other illicit drugs in various physical forms are primarily trafficked across major parts of the world through this route. The illicit substances can be analyzed using a number of techniques, e.g., microcrystalline test, TLC, HPLC, LC-MS, GC-MS, GC-FID, $\mathrm{CE}$, and FTIR.

\section{Case presentation}

In the current study, officials from a local law enforcement agency arrested an individual. The suspect intended to fly to Dubai from Allama Iqbal International Airport Lahore, Pakistan. He was carrying two packets of suspected orange colored tablets (called morphia tablets) in his suitcase. The total weight of seized morphia tablets was found to be $750 \mathrm{~g}$. The representative samples from both packets were submitted for analysis. The tablets were analyzed by using gas chromatography-mass spectrometry (GC-MS) according to SWGDRUG protocols (Comittee, 2001). The objective of the present study is to determine the physical characteristics of the tablets and to identify active constituents present in illicit "morphia tablets."

\section{Materials}

Morphia tablets seized by a law enforcement agency and later submitted at Narcotics Department, Punjab Forensic Science Agency, were used for the qualitative analysis. In this study, two packets of suspected tablets were seized and representative samples from both the packets were submitted to analyze for any possible illicit substance. The certified reference standards of heroin (diacetylmorphine), caffeine, and dextromethorphan were purchased from Sigma Aldrich (USA). Analytical grade methanol (MeOH) was purchased from Merck (USA). Statistical operations were performed on Microsoft Excel 2013.

\section{Instrumentation}

\section{Physical characteristics and presumptive testing}

Physical characteristics (i.e., shape, color, and embossing/ marking) of morphia tablets were observed macroscopically. Calibrated analytical balance ME104, Mettler Toledo (Ohio, US) was used for weighing. Thirty tablets from both of the samples were selected randomly and weighed. The presence of different types of illicit drugs was indicated by Chemical Spot Tests under SWGDRUG guidelines and recommended standard laboratory practices.

\section{Sample preparation for qualitative analysis on GC-MS}

In order to confirm the chemical composition of tablets in both the samples, five tablets from each sample were randomly selected for GC-MS analysis. A morphia tablet was ground to a fine homogeneous powder using neat and clean pestle mortar. Five (05) milligrams of sample was weighed on analytical balance and transferred to centrifuge tube. The sample was vortexed for $2 \mathrm{~min}$ with $2 \mathrm{~mL}$ of methanol and centrifuged at $1800 \mathrm{rpm}$ for $2 \mathrm{~min}$. The supernatant methanolic extract was transferred to a GC-MS vial by using a transfer pipette. The sample was run on GC with mass spectrometer detector (MSD) in scan mode.

\section{Gas chromatography - mass spectrometry (GC-MS)}

GC-MS (7890B-5977, GC System and MSD Triple Axis Detector, Agilent technologies, (Palo Alto, CA) with ALS (automated liquid sampler), was used for the analysis of aliquot from both of the samples. One microliter of the sample was injected in GC. Method parameters used for the analysis by GC-MS are given in Table 1 (Don Cooper, 2005). Results interpretation was performed on GC Chemstation (Agilent Technologies Co.) provided with NIST library. Major ion peaks were also compared with that of the certified reference standards.

\section{Results and discussion}

Morphia tablets are a rare product of clandestine laboratories, as these are prepared as a unit dosage like pharmaceutical tablets. Comprehensive physical and chemical 
Table 1 Analytical parameters of GC-MS

\begin{tabular}{|c|c|}
\hline & GC (7890B) \\
\hline Inlet & Split mode (50:1) \\
\hline Inlet temperature & $250^{\circ} \mathrm{C}$ \\
\hline Carrier gas & Helium (flow rate $1 \mathrm{~mL} / \mathrm{min}$ ) \\
\hline Inlet pressure & 14.69 psi \\
\hline Column & DB-5MS column $(20 \mathrm{~m} \times 180 \mu \mathrm{m} \times 0.18 \mu \mathrm{m})$ \\
\hline Oven program & $\begin{array}{l}70^{\circ} \mathrm{C}(2 \mathrm{~min}) \rightarrow 20^{\circ} \mathrm{C} / \mathrm{min} \rightarrow 200^{\circ} \mathrm{C}(0 \mathrm{~min}) \rightarrow \\
\left.7^{\circ} \mathrm{C} / \mathrm{min} \rightarrow 300^{\circ} \mathrm{C}(2 \mathrm{~min})\right) \rightarrow 25^{\circ} \mathrm{C} / \mathrm{min} \rightarrow \\
310^{\circ} \mathrm{C}(1 \mathrm{~min})\end{array}$ \\
\hline \multirow[t]{2}{*}{ Run time } & $26.186 \min$ \\
\hline & MS (5975C) \\
\hline Transfer line & $230^{\circ} \mathrm{C}$ \\
\hline Source temperature & $230^{\circ} \mathrm{C}$ \\
\hline Quad temperature & $150^{\circ} \mathrm{C}$ \\
\hline Mode & Scan \\
\hline Scan range & $43-550 \mathrm{~m} / \mathrm{z}$ \\
\hline
\end{tabular}

analysis of morphia tablets produced results that could be helpful to determine its comparative features to find source. Average weight of a tablet was determined. For sample A, it was found to be $58.24 \mathrm{mg}$ (with minimum weight $49.3 \mathrm{mg}$ of tablet and maximum weight $63.2 \mathrm{mg}$ of a tablet with a standard deviation of 3.20), and for sample B, it was $68.85 \mathrm{mg}$ (maximum $74.4 \mathrm{mg}$ and minimum $61.3 \mathrm{mg}$ with a standard deviation of 3.04). Both of the samples contained orange colored circular tablets with no embossing. Chemical spot tests indicated the presence of opiates in illicit tablets (Figs. 1 and 2). Analysis showed that the morphia tablets are a rare form of heroin. The morphia tablets vary greatly in color from street heroin powders seized in

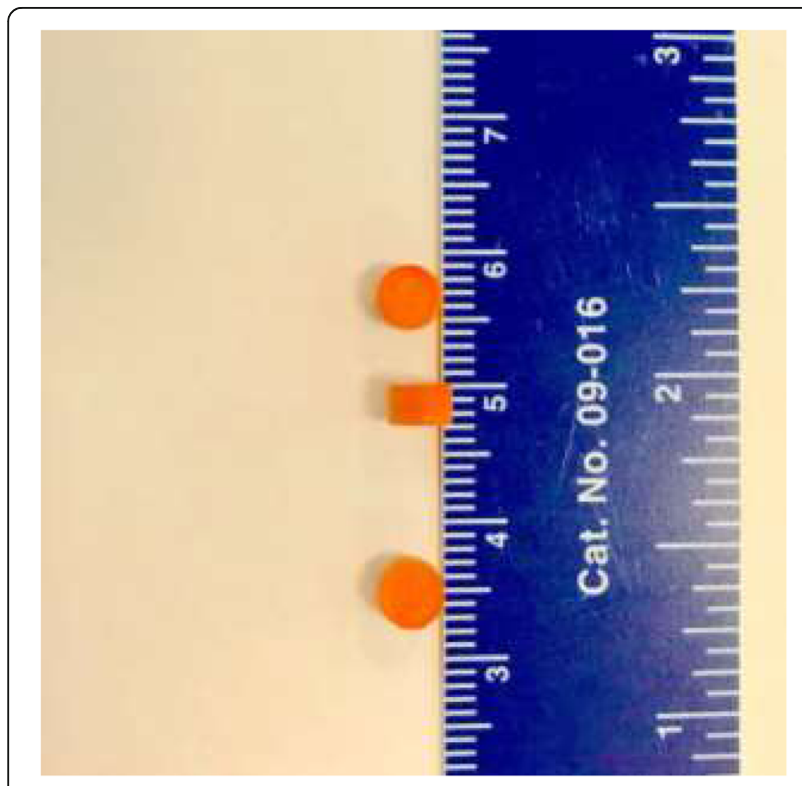

Fig. 1 Morphia tablets sample A

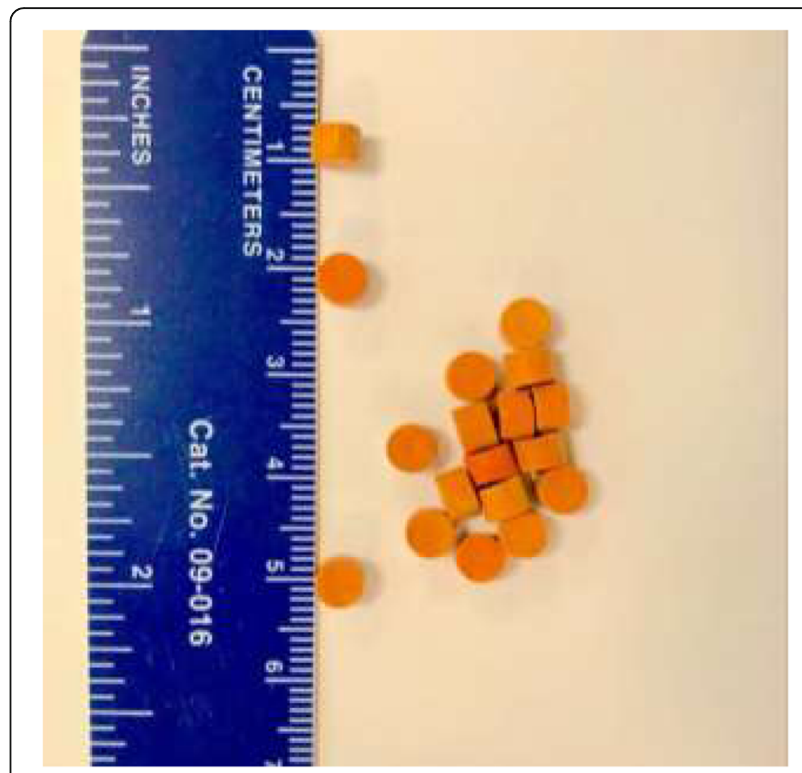

Fig. 2 Morphia tablets sample B

other parts of the world, i.e., brown powder in Switzerland (Briellmann et al., 2002); white, beige, and brown in Denmark (Kaa, 1994); white, brown, and gray powder in Egypt (Hassan, 2008); yellow, beige, and light brown in Kuwait (Alabkal et al., 2015); off-white powder in China (Guo et al., 2012); white, off-white, yellow, green, pink, and brown in Malaysia (Chan et al., 2012); brown powder, brown blocks (insoluble in water), and brown liquid in Iran (Akhgari et al., 2012).

Heroin is synthesized by the acetylation of morphine. Morphine is an opium alkaloid. The opium resin contains codeine, thebaine, papaverine, and noscapine as well. During heroin synthesis from opium resin, some intermediates or by-products like 6-acetylmorphine, 3-acetylmorphine, and acetylcodeine are also produced. The street heroin powder contains these intermediates and by-products as impurities due to poor purification procedure. The concentration of these alkaloids varies depending on the origin of heroin powder (Andreasen et al., 2009; Collins et al., 2007). The purity of heroin, by-products, impurities and concentration, and nature of adulterants varies from sample to sample in street heroin. The most common adulterants are caffeine, acetaminophen (Andreasen et al., 2009; Broséus et al., 2016a; Broséus et al., 2016b), dextromethorphan (Chan et al., 2012), phenobarbital, acetylthebaol, diazepam, clonazepam, pheniramine, biperiden, tramadol (Akhgari et al., 2012), chloroquine (Sulaiman et al., 2017) and lidocaine (Pichini et al., 2017). The qualitative analysis of morphia tablets using GC-MS showed that in addition to heroin tablets, sample A also contained caffeine, dextromethorphan, hydromorphone, and acetylcodiene, while sample B contained caffeine acetylcodiene, papaverine, and noscapine (Table 2). The confirmation of constituents of morphia 
Table 2 Results of qualitative analysis of "morphia tablets"

\begin{tabular}{|c|c|c|c|c|}
\hline Analyte & & Drug category & Retention time & Molecular ions (m/z) \\
\hline \multicolumn{5}{|c|}{ Sample A } \\
\hline 1. & Caffeine & Psychostimulants & 10.14 & $194,109,82,67,55,42,110$ \\
\hline 2. & Dextromethorphan & Non-opioid antitussive & 10.96 & $59,271,150,270,31,214,42,171$ \\
\hline 3. & Hydromorphone & Opium alkaloid & 12.84 & $285,96,229,228,70,214,115,200$ \\
\hline 4. & Acetylcodeine & Opium alkaloid derivative & 14.63 & $341,282,229,342,42,43,59,204$ \\
\hline 5. & Heroin & Opium alkaloid derivative & 15.79 & $327,43,369,268,310,42$ \\
\hline \multicolumn{5}{|c|}{ Sample B } \\
\hline 1. & Caffeine & Psychostimulants & 10.14 & $194,109,82,67,55,42,110$ \\
\hline 2. & Acetylcodeine & Opium alkaloid derivative & 14.57 & $341,282,229,342,42,43,59,204$ \\
\hline 3. & Heroin & Opium alkaloid derivative & 15.68 & $327,43,369,268,310,42$ \\
\hline 4. & Papaverine & Opium alkaloid & 18.45 & $339,324,338,325,340,308,154,292$ \\
\hline 5. & Noscapine & Opium alkaloid & 20.81 & $220,221,205,147,42,193,77,118$ \\
\hline
\end{tabular}

tablets was done by the comparison of retention time of samples peaks and mass spectrum with that of certified reference materials, NIST library, and reported literature. Major ions for heroin were 327, 43, 369, 268, 310, and 42; for caffeine 194, 109, 82, 67, 55, 195, 42, and 110; for dextromethorphan 59, 271, 150, 270, 31, 214, 42, and 17; for hydromorphone $285,96,229,228,70,214,115$, and 200; for acetylcodiene 341, 282, 229, 342, 42, 43, 59, and 204; for papaverine 339, 324, 338, 325, 340, 308, 154, and 292; and for noscapine (220, 221, 205, 147, 42, 193, 77, and 118) (Anthony C Moffat and Widdop, 2011). The results of qualitative analysis of two illicit morphia tablet samples using GC-MS are given in Table 2.

The concentration of heroin in illicit street powders varies from sample to sample. The gas chromatogram clearly shows the differences in the concentration of heroin in two analyzed samples, by means of peak height (Fig. 3 and 4). The concentration of heroin in "sample B" was much higher than the "sample A." The fluctuations in

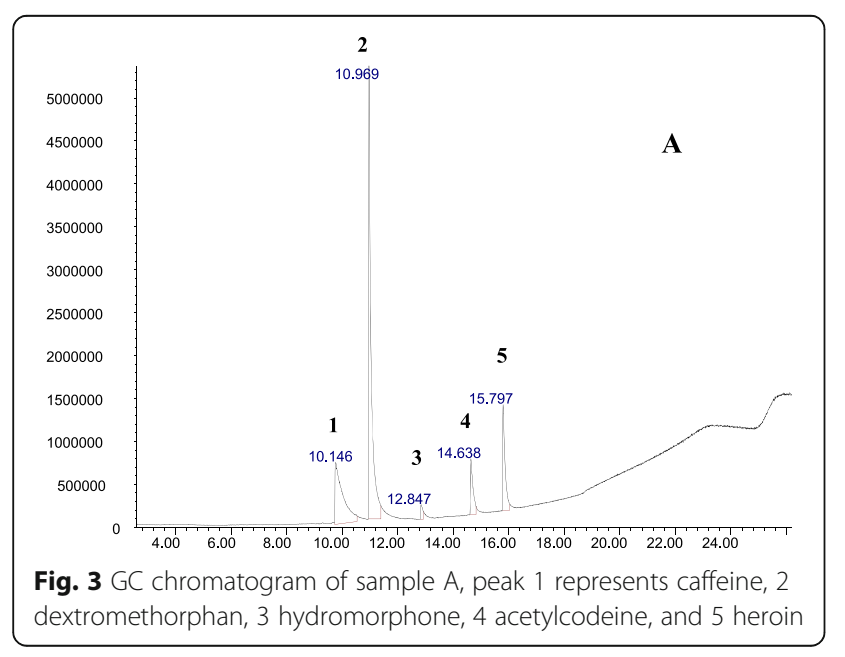

street heroin purity, presence of other psychotropic substances as adulterants, and sometimes combined abuse of other drugs with heroin can have fatal effects on abusers (Darke et al., 1999).

Seized morphia tablets are not influenced by trafficking, as it is not possible to add adulterants. External marks or stains may be left on tablets during trafficking. Tablets therefore only reflect the chemical synthesis procedure of the active component, diluents added, and the pressing (press marks, etc.) at clandestine laboratory. Because of no or very less effects of trafficking, tablets show the original physical and morphological characteristics as well as chemical information. The manufacturing process of tablets involves two steps, the synthesis, and the pressing. The concentration of heroin and other components in illicit morphia tablets is the same as manufactured. From intelligence and forensic point of view, complete drug profiling of tablets will help to determine the origin.

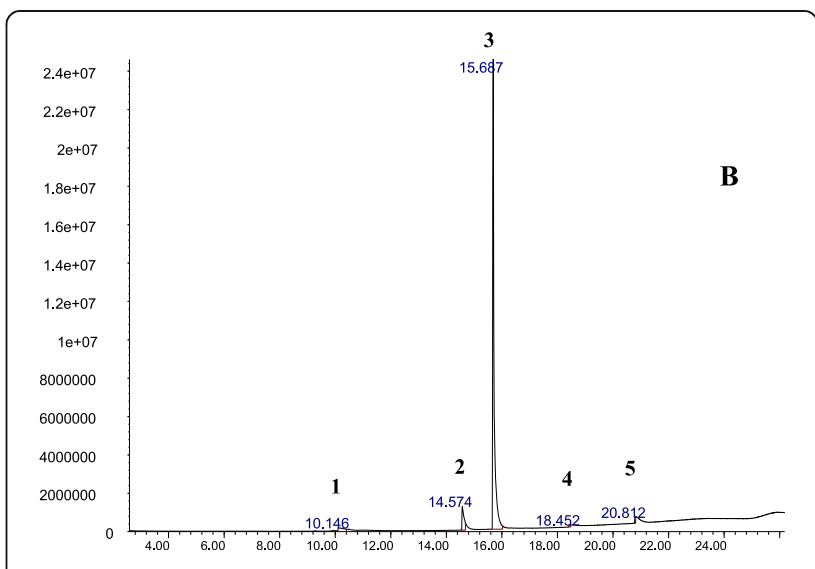

Fig. 4 GC chromatogram of sample A, peak 1 represents caffeine, 2 dextromethorphan, 3 heroin, 4 papaverine, and 5 noscapine 


\section{Conclusion}

The analysis of "morphia tablets" showed that these are composed of varied amounts of heroin and other components such as caffeine, acetylcodiene, dextromethorphan, papaverine, and noscapine. These tablets are merely the altered form of street heroin powder with different color and shape. In Pakistan, normally, the heroin is available as light brown powder while these circular orange tablets are some unique product. The possible reason for this change in color and physical form is to deceive the police and law enforcement agencies for safe and easy transportation of the drug. The variation in concentration of heroin in tablets can be fatal for abusers (Darke et al., 1999). The difference in chemical composition of samples suggested that these were from two different sources; however, the complete profiling of tablets is further suggested.

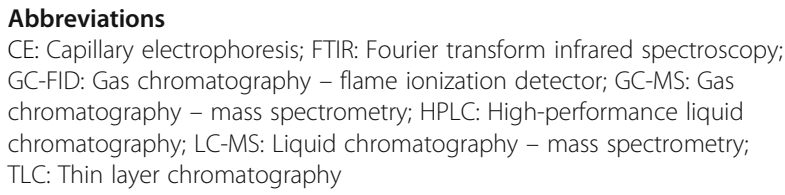

\section{Acknowledgements}

None

\section{Funding}

None

\section{Availability of data and materials}

Not available

\section{Authors' contributions}

$\mathrm{MU}$ is the main author and contributor of this article. TJ helped in GC-MS analysis, $A B$ helped in sampling and final editing of article, and $Y B$ helped GC-MS analysis and physical analysis, i.e., weight determination and photography. ZM, MS, SA, and MTC helped in writing. MS and MAT (DG PFSA) finally approved the article for publication. All authors read and approved the final manuscript.

\section{Author's information}

Muhammad Usman completed his MS Forensic Chemistry (2014) from Government College University Lahore and joined Punjab Forensic Science Agency (PFSA) in August 2014 as Junior Forensic Scientist at Narcotic Unit. At Narcotic Unit, PFSA Lahore, he is responsible for analysis of Narcotic Evidence (Seized Narcotic Drugs, e.g. hashish, cannabis, opium, heroin, poppy plants, amphetamine type stimulant, and narcotic tablets/injections) provided by Law Enforcement Agencies \& Court of Law and to testify in court as Expert Witness. He have analyzed and reported more than three thousand narcotic cases and reviewed almost two thousand cases. Furthermore, he has been engaged in conducting the research and interprets literature on criminal laboratory analytical methods, keeps current on techniques and trends, to research and develop new analytical methods and modification of already existing methods for better production of results. During his short professional career, he have attended various workshops and conferences at national and international level related to forensic science in order to get in touch the current progress in forensic science.

\section{Competing interests}

The authors declare that they have no competing interests.

\section{Publisher's Note}

Springer Nature remains neutral with regard to jurisdictional claims in published maps and institutional affiliations.

Received: 12 September 2018 Accepted: 5 December 2018 Published online: 22 December 2018

\section{References}

Akhgari M, Jokar F, Bahmanabadi L, Aleagha AE (2012) Street-level heroin seizures in Iran: a survey of components. J Subst Abus 17:348-355

Alabkal N, Metwally EK, Elnagdi M, Aldosery F, Abbas N (2015) Quantitative analysis of components of heroin seized in Kuwait by gas chromatography/ mass spectrometry. Am Chem Sci J 8:1-8

Andreasen MF, Lindholst C, Kaa E (2009) Adulterants and diluents in heroin, amphetamine, and cocaine found on the illicit drug market in Aarhus, Denmark. Open Forensic Sci J 2:16-20

Anthony C Moffat MDO, Widdop B (2011) Clarke's analysis of drug and poisons in pharmaceuticals, body fluids and postmortem materials, Fourth edn. Pharmaceutical Press, London

Briellmann TA, Dussy FE, Bovens MG (2002) Forensic analysis of heroin and cocaine seizures. CHIMIA Int J Chem 56:74-79

Broséus J, Baechler S, Gentile N, Esseiva P (2016a) Chemical profiling: a tool to decipher the structure and organisation of illicit drug markets: an 8-year study in Western Switzerland. Forensic Sci Int 266:18-28

Broséus J, Gentile N, Esseiva P (2016b) The cutting of cocaine and heroin: a critical review. Forensic Sci Int 262:73-83

Chan K-W, Tan G-H, Wong RC (2012) Investigation of illicit heroin seized in Malaysia: physical characteristics and chemical profiling. Aust J Forensic Sci 44:353-369

Collins M, Huttunen J, Evans I, Robertson J (2007) Illicit drug profiling: the Australian experience. Aust J Forensic Sci 39:25-32

Comittee S. (2001) Scientific Working Group for the Analysis of Seized Drugs (SWGDRUG), Recommendations for: Education and Training. Quality Assurance, Methods of Analysis, US Department of Justice, Drug Enforcement Administration/Executive Office of the President, Office ofNational Drug Control Policy, Counterdrug TechnologyAssessment Center

Darke S, Hall W, Weatherburn D, Lind B (1999) Fluctuations in heroin purity and the incidence of fatal heroin overdose. Drug Alcohol Depend 54:155-161

Don Cooper HN (2005) Methods for impurity profiling of heroin and cocaine, UNODC, Vienna, p 91

Emmanuel F, Attarad A (2006) Correlates of injection use of synthetic drugs among drug users in Pakistan: a case controlled study. J-Pak Med Assoc 56:119

Frank RS, Sobol SP (1990) Clandestine drug manufacturing laboratories, forensic science progress. Springer, pp 1-23

Guo Z, Zheng H, Lu Y, Wei Y (2012) Isolation and purification of heroin from heroin street samples by preparative high performance liquid chromatography. Forensic Sci Int 221:120-124

Hassan A.E.-A.G.A.A.E.-B.A.A. (2008) Chemical additives of street heroin in Cairo. Mansoura J Forensic Med Clin Toxicol. 14(1):1-12

Kaa E (1994) Impurities, adulterants and diluents of illicit heroin. Changes during a 12-year period. Forensic Sci Int 64:171-179

Pichini S, Busardò FP, Gregori A, Berretta P, Gentili S, Pacifici R (2017) Purity and adulterant analysis of some recent drug seizures in Italy. Drug Test Anal 9: 485-490

Sobol SP (2012) Clandestine drug manufacturing laboratories. Forensic Sci Progr 4:1

Sulaiman M, Kunalan V, Whei AYT, Lee WLJ, Yee JNJ, Xiong SLW, Bian CK (2017) Heroin in Malaysia and Singapore. Drug testing and analysis. 10(1):109-119

Syeda AF, Rasooly MH, Abidi SH, Modjarrad K, Ali S (2017) Opium trade and the spread of HIV in the Golden Crescent. Harm Reduct J 14. https://doi.org/10. 1186/s12954-017-0170-1 